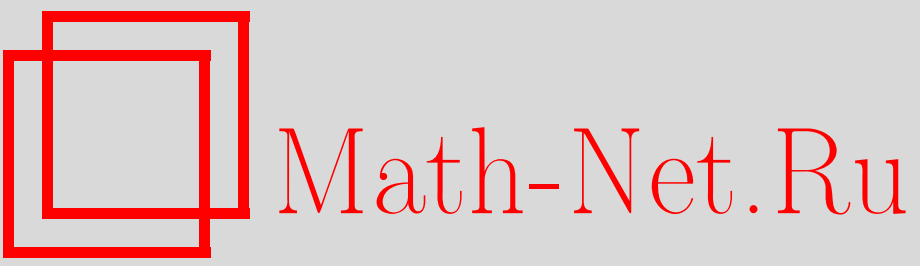

Е. А. Акимова, Об асимптотическом поведении локально периодической тонкой композитной пластины, УМН, 2004, том 59, выпуск 5, 143-144

DOI: https://doi.org/10.4213/rm775

Использование Общероссийского математического портала Math-Net.Ru подразумевает, что вы прочитали и согласны с пользовательским соглашением

http://www.mathnet.ru/rus/agreement

Параметры загрузки:

IP : 18.208 .226 .222

26 апреля 2023 г., 16:44:51 


\title{
ОБ АСИМПТОТИЧЕСКОМ ПОВЕДЕНИИ ЛОКАЛЬНО ПЕРИОДИЧЕСКОЙ ТОНКОЙ КОМПОЗИТНОЙ ПЛАСТИНЫ
}

\author{
Е. А. АкимовА
}

Пусть $\omega \subset \mathbb{R}^{2}$ - область, ограниченная простьм замкнутым контуром $\partial \omega, P=\{\xi=(\eta, \zeta) \in$ $\left.\mathbb{R}^{3}: 0 \leqslant \eta_{1} \leqslant l_{1}, 0 \leqslant \eta_{2} \leqslant l_{2}\right\}$ - призма с прямоуголшным основанием и $\varphi_{y}: P \rightarrow P$ - семейство диффеоморфизмов, гладко зависящих от точки $y=\left(y_{1}, y_{2}\right)$. Введем замкнутое множество $S$, расположенное в $P$, такое, что множество $\Pi_{h}=\left\{x: h^{-1} x \in S(y), y \in \mathbb{R}^{2}\right\}$ является связным (здесь $S(y)=\varphi_{y} S$ ). Пластину $\Omega_{h}$ и ее боковую поверхность $\Gamma_{h}$ введем с помощью формул $\Omega_{h}=\left\{x=(y, z): y \in \omega, \frac{x}{h} \in S(y)\right\}, \Gamma_{h}=\left\{x: y \in \partial \omega, \frac{x}{h} \in S(y)\right\}, h \in(0,1]$-малшій параметр. Рассмотрим смешанную краевую задачу теории упругости

$$
\begin{gathered}
D\left(-\nabla_{x}\right)^{\top} A(\xi, y) D\left(\nabla_{x}\right) u(h, x)=f(h, x), \quad x \in \Omega_{h}, \\
D(n)^{\top} A(\xi, y) D\left(\nabla_{x}\right) u(h, x)=g(h, x), \quad x \in \partial \Omega_{h} \backslash \Gamma_{h}, \\
u(h, x)=0, \quad x \in \Gamma_{h},
\end{gathered}
$$

где $u\left(u_{1}, u_{2}, u_{3}\right)^{\top}$ - вектор смещений; $A$-положительно определенная симметрическая матрица упругих модулей материала пластины, зависящая от медленных $y$ и быстрых $\xi=\left(\eta_{1}, \eta_{2}, \zeta\right)=$ $\left(\frac{y_{1}}{h}, \frac{y_{2}}{h}, \frac{z}{h}\right)$ переменных; $f$ и $g$ - столбцы объемных сил и поверхностных усилий; $n$ - единичный вектор внешней нормали к поверхности $\Sigma=\partial \Omega_{h} \backslash \Gamma_{h} ; D\left(\nabla_{x}\right)$ - матрица дифференциальных операторов

$$
D\left(\nabla_{x}\right)=\left(\begin{array}{cccccc}
\partial_{1} & 0 & \alpha \partial_{2} & \alpha \partial_{z} & 0 & 0 \\
0 & \partial_{2} & \alpha \partial_{1} & 0 & \alpha \partial_{z} & 0 \\
0 & 0 & 0 & \alpha \partial_{1} & \alpha \partial_{2} & \partial_{z}
\end{array}\right)^{\top}, \quad \partial_{i}=\frac{\partial}{\partial y_{i}}, \quad \partial_{z}=\frac{\partial}{\partial z}, \quad \alpha=\frac{1}{\sqrt{2}}
$$

Примем следующие асимптотические анзацы для правых частей и для решения задачи (1)-(3):

$$
\begin{gathered}
f(h, x)=h^{-1} f^{0}(\xi, y)+h^{0} \bar{f}^{0}(y)+\tilde{f}(h, x), \\
g(h, y)=h^{0} g^{0}(\xi, y)+\tilde{g}(h, x), \\
h^{-2} U^{-2}(y)+h^{-1} U^{-1}(\xi, y)+h^{0} U^{0}(\xi, y)+h^{1} U^{1}(\xi, y)+\cdots .
\end{gathered}
$$

Члены $f^{0}, g^{0}, \bar{f}^{0}$ анзацев подчиним соотношениям

$$
\int_{S(y)} f_{3}^{0} d \xi+\int_{\Sigma(y)} g_{3}^{0} d s_{\xi}=0, \quad \bar{f}^{0}(y)=e^{3} \bar{f}_{3}^{0}(y)
$$

Подставив разложения (4)-(6) в уравнения (1)-(2), получим некие равенства, которьм функции $U^{-2}$ и $U^{-1}$ удовлетворяют автоматически, а разрешимость задач на $U^{0}$ и $U^{1}$ доказана, например, в [1] (см. предложение 2.5.5(2), лемму 6.4.1 и теорему 4.1.4).

С помощью весового анизотропного неравенства Корна (см. [2]) для локально периодической пластины $\Omega_{h}:\left|u ; \Omega_{h}\right|^{2} \leqslant c \mathscr{E}(u ; \Omega)$, в котором $u \in \stackrel{\circ}{H^{1}}\left(\Omega_{h}, \Gamma_{h}\right)^{3}, c$ не зависит от поля $u$ и параметра $h$,

$$
\begin{aligned}
&\left|u ; \Omega_{h}\right|^{2}=\int_{\Omega_{h}}\left\{\sum_{i=1}^{2}\left[\left|\nabla_{y} u_{i}\right|^{2}+h^{2} \rho_{h}^{-2}\left(\left|\frac{\partial u_{i}}{\partial z}\right|^{2}+\left|\frac{\partial u_{3}}{\partial y_{i}}\right|^{2}\right)+\rho_{h}^{-2}\left|u_{i}\right|^{2}\right]\right. \\
&\left.+\left|\partial_{z} u_{3}\right|^{2}+h^{2} \rho_{h}^{-4}\left|u_{3}\right|^{2}\right\} d x, \quad \rho_{h}(x)=h+\operatorname{dist}\left\{x, \Gamma_{h}\right\}
\end{aligned}
$$

доказьвается следующая теорема об асимптотике. 
TeOpema 1. Пусть $f^{0} \in H^{1+\gamma}\left(\omega \rightarrow C_{\mathrm{per}}^{0, \beta}(S(y))^{3}\right), g^{0} \in H^{1+\gamma}\left(\omega \rightarrow C_{\mathrm{per}}^{1, \beta}(\Sigma(y))^{3}\right)$, $\bar{f}_{3}^{0} \in H^{\gamma}(\omega), \tilde{f} \in L_{2}\left(\Omega_{h}\right)^{3}, \tilde{g} \in L_{2}\left(\Sigma_{h}\right)^{3}$. Здесь $H^{1+\gamma}(\omega \rightarrow \ldots)$ - пространство Соболева-Слободецкого абстрактных функций с нормой

$$
\begin{aligned}
& \left\|\left\{f^{0}, g^{0}\right\} ; H^{1+\gamma}(\omega \rightarrow \ldots)\right\|=\left[\int _ { \omega } \left(\left\|\left\{f^{0}(y, \cdot), g^{0}(y, \cdot)\right\} ; \ldots\right\|^{2}\right.\right. \\
& \left.\quad+\left\|\left\{\nabla_{y} f^{0}(y, \cdot), \nabla_{y} g^{0}(y, \cdot)\right\} ; \ldots\right\|^{2}\right) d y+\int_{\omega} \int_{\omega} \|\left\{\left(\nabla_{y} f^{0}\right)(y, \cdot)\right. \\
& \left.\left.\quad-\left(\nabla_{y} f^{0}\right)(\mathbf{y}, \cdot),\left(\nabla_{y} g^{0}\right)(y, \cdot)-\left(\nabla_{y} g^{0}\right)(\mathbf{y}, \cdot)\right\} ; \ldots \|^{2}|y-\mathbf{y}|^{-2(1+\gamma)} d y d \mathbf{y}\right]^{1 / 2},
\end{aligned}
$$

многоточие заменяет область значений, в качестве которой берутся подпространства $C_{\operatorname{per}}^{0, \beta}(S(y))$ и $C_{\text {per }}^{1, \beta}(\Sigma(y))$ функций гёльдеровских классов $C^{0, \beta}(S(y))$ и $C^{1, \beta}(\Sigma(y))$, удовлетворяющих условиям периодичности

$$
\left.U\right|_{\varphi_{y}\left(\eta_{i}=0\right)}=\left.U\right|_{\varphi_{y}\left(\eta_{i}=l_{i}\right)},\left.\quad \frac{\partial U}{\partial \eta_{i}}\right|_{\varphi_{y}\left(\eta_{i}=0\right)}=\left.\frac{\partial U}{\partial \eta_{i}}\right|_{\varphi_{y}\left(\eta_{i}=l_{i}\right)}, \quad i=1,2,
$$

или берется прямое произведение $C_{\mathrm{per}}^{0, \beta}(S(y)) \times C_{\mathrm{per}}^{1, \beta}(\Sigma(y))$. Норма в $C_{\mathrm{per}}^{s, \beta}(S(y))$ задается формулой

$$
\begin{aligned}
\left\|v ; C_{\mathrm{per}}^{s, \beta}(S(y))\right\|= & \sum_{k=0}^{s} \sup \left\{\left|\nabla_{x}^{k} v(x)\right| ; x \in S(y)\right\} \\
& \quad+\sup \left\{|x-\mathbf{x}|^{-\beta}\left|\left(\nabla_{x}^{s} v\right)(x)-\left(\nabla_{x}^{s} v\right)(\mathbf{x})\right| ; x, \mathbf{x} \in S(y)\right\},
\end{aligned}
$$

а норма в $C_{\mathrm{per}}^{s, \beta}(\Sigma(y))$ получается из (7) заменой $S(y)$ на $\Sigma(y)$ и $\nabla_{x}$ на касательный градиент $\nabla_{\tau}$. Тогда решение и задачи (1)-(3) и его асимптотическое приближение $\bar{u}^{0}(h, x)=h^{-2} U^{-2}(y)+h^{-1} U^{-1}(\zeta, y)+h^{0} U^{0}(\xi, y)$ связаны неравенством

$$
\left|u-\bar{u}^{0} ; \Omega_{h}\right|+\left\|D\left(\nabla_{x}\right)(u)-D\left(\nabla_{x}\right)\left(\bar{u}^{0}\right) ; L_{2}\left(\Omega_{h}\right)\right\| \leqslant c h^{\gamma-1 / 2}\left(\mathscr{N}_{1+\gamma}+\widetilde{\mathscr{N}}_{\gamma-1 / 2}\right),
$$

где

$$
\begin{gathered}
\widetilde{N}_{\gamma-1 / 2}=h^{1 / 2-\gamma}\left[\sum_{i=1}^{2}\left(\left\|\rho_{h} \tilde{f}_{i} ; L_{2}\left(\Omega_{h}\right)\right\|+h^{-1 / 2}\left\|\rho_{h} \tilde{g}_{i} ; L_{2}\left(\Sigma_{h}\right)\right\|\right)\right. \\
\left.+h^{-1}\left\|\rho_{h}^{2} \tilde{f}_{3} ; L_{2}\left(\Omega_{h}\right)\right\|+h^{-3 / 2}\left\|\rho_{h}^{2} \tilde{g}_{3} ; L_{2}\left(\Sigma_{h}\right)\right\|\right] \\
\mathscr{N}_{1+\gamma}=\left\|\vec{f}_{3}^{0} ; H^{\gamma}(\omega)\right\|+\left\|\left\{f^{0}, g^{0}\right\} ; H^{1+\gamma}\left(\omega \rightarrow C_{\mathrm{per}}^{0, \beta}(S(y))^{3} \times C_{\mathrm{per}}^{1, \beta}(\Sigma(y))^{3}\right)\right\| ;
\end{gathered}
$$

постоянная с не зависит ни от $h \in(0,1]$, ни от составляющих $f^{0}, g^{0}, \bar{f}^{0}$ и $\tilde{f}, \tilde{g}$ правых частей задачи.

Резулштаты, приводимые в данной работе, получены совместно с Г. А. Чечкиным и С. А. Назаровым.

\section{СПИСОК ЛИТЕРАТУРЫ}

[1] С. А. Назаров. Асимптотическая теория тонких пластин и стержней. Т. 1. Понижение размерности и интегральные оценки. Новосибирск: Научная книга, 2002. [2] Е. А. Акимова, С. А. Назаров, Г. А. Чечкин // Докл. РАН. 2001. Т. 380. № 4. С. 439-442.

Московский государственньй

Принято редколлегией университет им. М. В. Ломоносова 23.07 .2004

E-mail: akimova_ea@mail.ru 